\title{
Relating Breakup and Incomplete Fusion of Weakly Bound Nuclei through a Classical Trajectory Model with Stochastic Breakup
}

\author{
A. Diaz-Torres, ${ }^{1}$ D. J. Hinde, ${ }^{1}$ J. A. Tostevin, ${ }^{1,2}$ M. Dasgupta, ${ }^{1}$ and L. R. Gasques ${ }^{1}$ \\ ${ }^{1}$ Department of Nuclear Physics, Research School of Physical Sciences and Engineering, Australian National University, \\ Canberra, ACT 0200, Australia \\ ${ }^{2}$ Department of Physics, University of Surrey, Guildford, GU2 7XH, United Kingdom \\ (Received 18 September 2006; published 11 April 2007)
}

\begin{abstract}
A classical dynamical model that treats breakup stochastically is presented for low energy reactions of weakly bound nuclei. The three-dimensional model allows a consistent calculation of breakup, incomplete, and complete fusion cross sections. The model is assessed by comparing the breakup observables with continuum discretized coupled-channel quantum mechanical predictions, which are found to be in reasonable agreement. Through the model, it is demonstrated that the breakup probability of the projectile as a function of its distance from the target is of primary importance for understanding complete and incomplete fusion at energies near the Coulomb barrier.
\end{abstract}

DOI: 10.1103/PhysRevLett.98.152701

PACS numbers: 25.70.Jj, 25.70.Mn

Recent developments of radioactive isotope accelerators provide an opportunity to investigate on Earth the fusion reactions that form heavy elements in the cosmos. These involve reactions of nuclei far from stability, the most exotic of which are often very weakly bound. Breakup of weakly bound nuclei is thus an important process in their collisions with other nuclei. A major consequence of breakup is that not all the resulting breakup fragments might be captured by the target, termed incomplete fusion (ICF); capture of the entire projectile by the target is called complete fusion (CF). Such ICF processes can dramatically change the nature of the reaction products, as has been investigated in detail for the stable weakly bound nuclei ${ }^{9} \mathrm{Be}$ and ${ }^{6,7} \mathrm{Li}[1]$. There, at energies above the fusion barrier, CF yields were found to be only $\sim 2 / 3$ of those expected, the remaining $1 / 3$ being in ICF products. Events where the projectile breaks up and none of the fragments are captured provide an important diagnostic of the reaction dynamics. This we call no-capture breakup (NCBU), also referred to as elastic breakup.

In a conventional picture of fusion, two colliding nuclei will fuse if they overcome the potential barrier due to their mutual Coulomb and nuclear interactions. The additional breakup degrees of freedom when one of the colliding nuclei is weakly bound makes the process very much more complicated. An outstanding theoretical challenge is to model the CF and ICF processes in such collisions, since this separation is crucial to understand the effects of breakup on fusion [1,2]. Quantum mechanical few-body approaches, such as the continuum discretized coupledchannel (CDCC) method [3,4] and the time-dependent wave packet method [5], cannot separate incomplete and complete fusion contributions to their absorptive cross sections [6], since both result in depletion of the total few-body wave function. The CDCC method can, however, make reliable predictions of the NCBU process [7], as will be exploited here. What, then, are the alternatives to the above models? A novel optical decoherence model has been suggested [6] but has yet to be implemented. Another approach is to use the concept of classical trajectories which allow CF and ICF events to be separated, as in the two-dimensional model of Ref. [8]. Classical dynamical models are also being successfully applied to describe atomic many-body collisions, including ionization [9], a process in some ways analogous to breakup.

In this Letter we present a new three-dimensional classical dynamical reaction model that allows quantitative calculations of CF and ICF yields above the barrier. This is achieved by the introduction of a stochastically sampled breakup function. Crucially, this function can be determined from experimental sub-barrier breakup data [10] or from CDCC calculations. We apply the model to twobody projectiles, although it can be extended to more complex many-body breakup scenarios. As individual trajectories are followed, the model allows a clear identification of CF, ICF, and NCBU events, allowing predictions of their yields as well as breakup fragment angular, kinetic energy, and relative energy distributions. This unique capability provides a powerful tool for interpreting future fusion experiments with radioactive nuclei and in applications to $\gamma$ ray spectroscopy [11]. The model is assessed quantitatively by comparing its predictions for NCBU observables with those of fully quantum mechanical CDCC calculations. These are carried out for a simplified test case. The classical model $\mathrm{CF}$ and ICF predictions are then compared to experimental data.

The classical model calculations are as follows. The weakly bound (two-body) projectile $P$, with incident energy $E_{0}$ and orbital angular momentum $L_{0}$, is incident on the $\operatorname{target} T$, initially at rest in the laboratory frame. Prior to breakup the projectile follows a classical orbit with a $P-T$ distance of closest approach $R_{\min }\left(E_{0}, L_{0}\right)$. These orbits of the bound projectile are calculated by numerical solution of the classical equations of motion in the presence of the 
Coulomb and nuclear potentials between $P$ and $T$. These generate a Coulomb barrier for head-on $\left(L_{0}=0\right)$ collisions of height $V_{B}^{P T}$ at a separation $R_{B}^{P T}$.

The effects of the Coulomb and nuclear two-body interactions that cause breakup are encoded in a local breakup probability $\mathcal{P}_{\mathrm{BU}}^{L}(R)$, a function of the projectile-target separation $R$, such that $\mathcal{P}_{\mathrm{BU}}^{L}(R) d R$ is the probability of breakup on the interval $R$ to $R+d R$. Crucially, for a given projectile-target combination, both experimental data [10] and $\mathrm{CDCC}$ calculations indicate that the integral of this breakup probability along a given classical orbit is an exponential function of its distance of closest approach, $R_{\text {min }}\left(E_{0}, L_{0}\right)$. Explicitly,

$$
P_{\mathrm{BU}}\left(R_{\mathrm{min}}\right)=2 \int_{R_{\min }}^{\infty} \mathcal{P}_{\mathrm{BU}}^{L}(R) d R=A \exp \left(-\alpha R_{\min }\right),
$$

from which it follows uniquely that the local function has the same exponential form, $\mathcal{P}_{\mathrm{BU}}^{L}(R) \propto \exp (-\alpha R)$.

For each $L_{0}$ (chosen to be an integer number of $\hbar$ ) a sample of $N$ incident projectiles is taken. The position of breakup on this orbit is determined by sampling a breakup radius $R_{\mathrm{BU}}$ on the interval $\left[R_{\min }\left(E_{0}, L_{0}\right), \infty\right]$ with the weighting $\mathcal{P}_{\mathrm{BU}}^{L}(R)$. Although dependent on the constants $A$ and $\alpha$, the exponential weighting of $\mathcal{P}_{\mathrm{BU}}^{L}(R)$ will clearly place most $R_{\mathrm{BU}}$ in the vicinity of $R_{\min }$. If the chosen $L_{0}$ is less than the critical partial wave for projectile fusion, $L_{\mathrm{cr}}$, then the associated trajectory would normally lead to CF, i.e., $R_{\text {min }} \leq R_{B}^{P T}$. For these $L_{0}$ we set $R_{\text {min }}=R_{B}^{P T}$, when sampling $R_{\mathrm{BU}}$, and all breakup events are confined to the incoming branch of the projectile trajectory. For $L_{0}>L_{\mathrm{cr}}$ breakup can take place on both the entrance and the exit branches of the classical orbit, which are sampled equally. Having chosen the position with the projectile center of mass at breakup, it is instantaneously broken up into fragments $\mathrm{F} 1$ and $\mathrm{F} 2$.

Following breakup, fragments F1 and F2 now interact with $T$ and with each other via real central two-body potentials having Coulomb barriers $V_{B}^{i j}$ at separations $R_{B}^{i j}$, $i, j=1,2, T, i \neq j$. The instantaneous dynamical variables of the excited projectile at breakup, its total internal energy $\varepsilon_{12}$, angular momentum $\vec{\ell}_{12}$, and the separation of the fragments $\vec{d}_{12}$ are all Monte Carlo sampled. The initial separations $d_{12}$ between the fragments are Gaussian distributed in their classically allowed region to mimic the radial probability distribution of the projectile ground-state (GS) wave function. For high $\ell_{12}$ excitations, when there is no barrier between F1 and F2, $d_{12}$ is equated with their external turning point. In the test calculation below, the orientation of $\vec{d}_{12}$ is chosen randomly over the $4 \pi$ solid angle, while the orientation of $\vec{\ell}_{12}$ is chosen randomly from all directions orthogonal to $\vec{d}_{12}$. Other distributions could easily be encoded in cases where the two breakup fragments are not identical. $\ell_{12}$ is sampled uniformly on the interval $\left[0, \ell_{\max }\right]$. For $\varepsilon_{12}$ we tested two sampling functions for energies between the top of the barrier, $V_{B}^{12}$, and a chosen maximum $\varepsilon_{\max }$. Using uniform weighting or an exponentially decreasing weighting yielded very similar outcomes, although the convergence of the observables was faster for the latter, which was therefore used. Both $\ell_{\max }$ and $\varepsilon_{\max }$ were increased until convergence of the observables occurred, as in the CDCC calculations.

Having fixed the positions and dynamical variables of the excited projectile fragments at the moment of breakup, the instantaneous velocities of the particles F1, F2, and $T$ are dictated by conservation of energy, linear momentum, and angular momentum in the overall center of mass frame of the projectile and target system. These breakup initial conditions were transformed to the laboratory frame where the equations of motion are solved. The computed trajectories of F1, F2, and $T$ determine the number of ICF, CF, and NCBU events, fragment $F j$ being assumed to be captured if the classical trajectories take it within the fragment-target barrier radius $R_{B}^{j T}$.

From the $N$ breakup events sampled for each projectile angular momentum $L_{0}$, the numbers of events $N_{i}$ in which $i=0$ (NCBU), 1 (ICF), or 2 (CF) fragments are captured determine the relative yields $\tilde{P}_{i}=N_{i} / N$ of these three processes after breakup, with $\tilde{P}_{0}+\tilde{P}_{1}+\tilde{P}_{2}=1$. The absolute probabilities $P_{i}\left(E_{0}, L_{0}\right)$ of the three reaction processes are determined from the relative yields and the integrated breakup probability over the whole trajectory $P_{\mathrm{BU}}\left(R_{\min }\right)$ :

$$
\begin{gathered}
P_{0}\left(E_{0}, L_{0}\right)=P_{\mathrm{BU}}\left(R_{\min }\right) \tilde{P}_{0}, \\
P_{1}\left(E_{0}, L_{0}\right)=P_{\mathrm{BU}}\left(R_{\min }\right) \tilde{P}_{1}, \\
P_{2}\left(E_{0}, L_{0}\right)=\left[1-P_{\mathrm{BU}}\left(R_{\min }\right)\right] H\left(L_{\mathrm{cr}}-L_{0}\right) \\
+P_{\mathrm{BU}}\left(R_{\min }\right) \tilde{P}_{2},
\end{gathered}
$$

where $H(x)$, the Heaviside step function, is unity for $x \geq 0$. Finally, the physical cross sections are calculated using

$$
\sigma_{i}\left(E_{0}\right)=\pi \lambda^{2} \sum_{L_{0}}\left(2 L_{0}+1\right) P_{i}\left(E_{0}, L_{0}\right),
$$

where $\lambda^{2}=\hbar^{2} /\left[2 m_{P} E_{0}\right]$ and $m_{P}$ is the projectile mass. Other observables, e.g., the angle, kinetic energy, and relative energy distributions of the fragments from NCBU events, are calculated by tracking their trajectories to a large distance from the target $(200 \mathrm{fm}$ in the calculations presented below).

In order to validate our approach, NCBU observables, reliably calculated within the CDCC quantum mechanical approach, are compared with the classical model calculation. We do so in a simplified model case for the reaction of a pseudo- ${ }^{8} \mathrm{Be}$ projectile $P$ (assuming a weakly bound state of two $\alpha$ particles) with a ${ }^{208} \mathrm{~Pb}$ target. The CDCC and classical model calculations are matched as closely as possible, as discussed below. 
We bind $P$ in a nodeless $s$ state with a separation energy of $0.5 \mathrm{MeV}$ in the Woods-Saxon (WS) potential well $(V, r, a) \equiv(16.7 \mathrm{MeV}, 1.20 \mathrm{fm}, 0.62 \mathrm{fm})$. The same potential was used to construct the continuum states of $P$, which are nonresonant in all partial waves. The Coulomb potential between all particles is that of uniformly charged spheres with a radius parameter $1.2 \mathrm{fm}$. The CDCC breakup model space includes (i) even $\ell_{12}$ up to $4 \hbar$, (ii) a maximum $\alpha-\alpha$ relative energy of $12 \mathrm{MeV}$, divided into 12 bins in each partial wave, and (iii) $\alpha-T$ potential multipoles up to quadrupole terms. The $\alpha-T$ optical potential is the real WS interaction $(62 \mathrm{MeV}, 1.39 \mathrm{fm}, 0.62 \mathrm{fm})$. A diagonal absorptive WS potential $(10 \mathrm{MeV}, 1.30 \mathrm{fm}$, $0.74 \mathrm{fm}$ ) is included in all channels, its range chosen so that absorption occurs inside the fusion radius of the $\alpha-T$ system, $R_{B}^{\alpha T}=10.6 \mathrm{fm}$. Calculations were carried out at a laboratory energy of $E_{0}=45 \mathrm{MeV}$.

A critical input to the classical model is the local projectile breakup probability $\mathcal{P}_{\mathrm{BU}}^{L}(R)$ at a separation $R$ from the target, deduced from $P_{\mathrm{BU}}\left(R_{\min }\right)$. To allow comparison with the CDCC, $P_{\mathrm{BU}}\left(R_{\min }\right)$ has been deduced from the CDCC breakup cross sections, $\sigma_{J}(\mathrm{NCBU})$, for each $P-T$ partial wave. This is well represented by an exponential function (beyond the strongly absorbed partial waves), with $A=1.38$ and $\alpha=0.293 \mathrm{fm}^{-1}$ [see Eq. (1)]. Experimental parameters defining $P_{\mathrm{BU}}\left(R_{\min }\right)$ have been deduced from breakup measurements for the reaction ${ }^{9} \mathrm{Be}+{ }^{208} \mathrm{~Pb}$ for a wide range of sub-barrier energies [10]. The parameters which best describe the experimental NCBU yields are $A=1.68 \times 10^{4}$ and $\alpha=0.922 \mathrm{fm}^{-1}$. The CDCC (dashed line) and empirical $P_{\mathrm{BU}}\left(R_{\min }\right)$ (thick solid line) distributions are compared in Fig. 1. It is significant that the two functions are very different, the slope parameter $\alpha$ depending sensitively on the reaction and the breakup mechanism. For instance, our model of ${ }^{8} \mathrm{Be}$ breakup, unlike the physical ${ }^{9} \mathrm{Be}$, does not generate Coulomb or nuclear dipole breakup couplings. Nor does

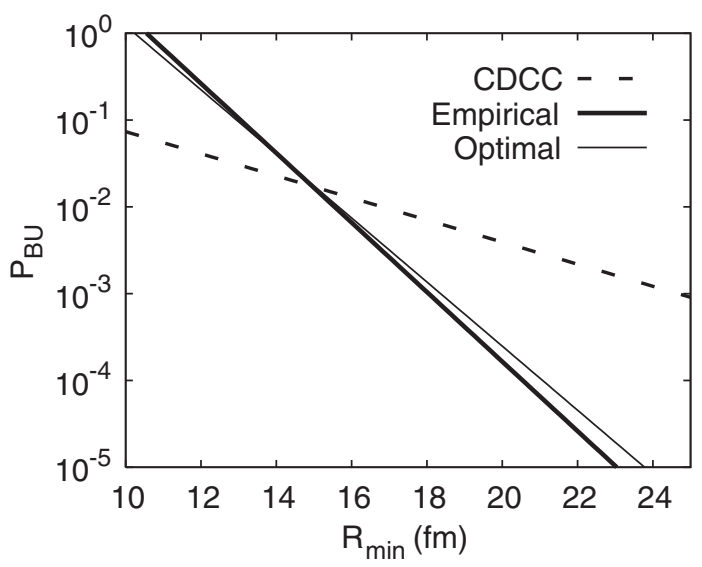

FIG. 1. The $P_{\mathrm{BU}}$ vs $R_{\min }$ from the CDCC model, the empirical line from the ${ }^{9} \mathrm{Be}+{ }^{208} \mathrm{~Pb}$ sub-barrier data [10], and the optimal function used in Fig. 3. it include the effect of neutron transfer channels that may be significant in the empirical distribution [12]. This comparison shows that the $\mathcal{P}_{\mathrm{BU}}^{L}(R)$ have high diagnostic value, and many reaction observables are also sensitive to the assumed $\mathcal{P}_{\mathrm{BU}}^{L}(R)$, as shown below.

We can now use the CDCC-derived $\mathcal{P}_{\mathrm{BU}}^{L}(R)$ as the sampling distribution in the stochastic classical model calculations to assess the consistency of the observables calculated with the quantum mechanical and classical dynamical models. In sampling breakup $\ell_{\max }=4 \hbar$ and $\varepsilon_{\max }=6 \mathrm{MeV}$. Guided by the calculated GS probability of the projectile, the Gaussian distribution of $d_{12}$ values is taken to have a centroid and variance of $1.8 \mathrm{fm}$ and $1.0 \mathrm{fm}$, respectively. The same real potentials are used as in the CDCC when solving the post-breakup three-body problem. The potential used for the $P\left({ }^{8} \mathrm{Be}\right)-T$ system prior to breakup, (120.9 MeV, $1.39 \mathrm{fm}, 0.76 \mathrm{fm})$, is consistent with the folding model potential derived from the $\alpha-T$ potentials and the GS wave function of $P$. In this way the extended size and weak binding of $P$ are taken into account prior to breakup. Values of $L_{0} \leq 100 \hbar$ were found to be sufficient for the convergence of the observables.

The calculated total absorption and total NCBU cross sections from the CDCC are $\sigma(\mathrm{abs})=327 \mathrm{mb}$ and $\sigma(\mathrm{NCBU})=97 \mathrm{mb}$, compared with $\sigma(\mathrm{abs})=300 \mathrm{mb}$ and $\sigma(\mathrm{NCBU})=93 \mathrm{mb}$ from the classical model. Figure 2 compares the CDCC (solid curves) and the classical model (dashed curves) for (a) the breakup cross sections as a function of $P-T$ partial wave, (b) the singles $\alpha$-particle angular distribution, (c) the $\alpha$ - $\alpha$ relative energy distribution, and (d) the singles $\alpha$-particle energy distribution. The classical and CDCC results are seen to agree
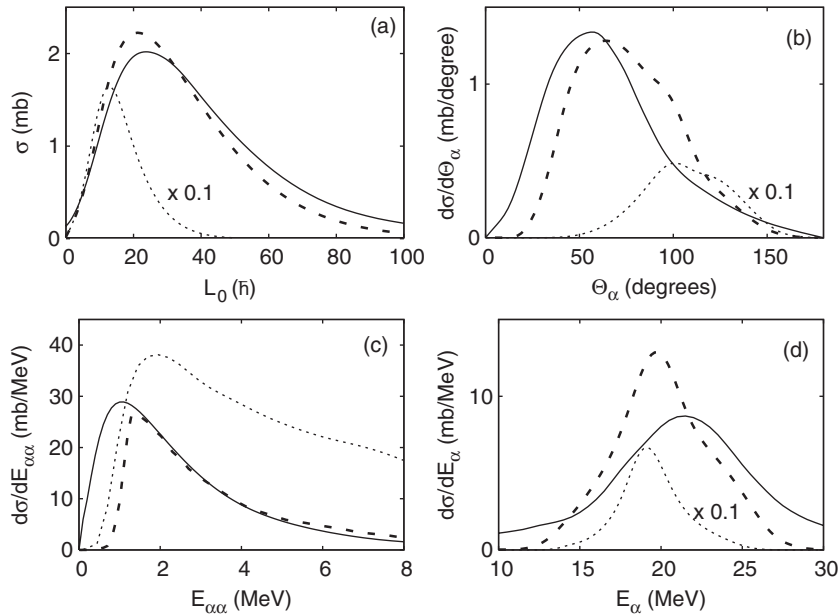

FIG. 2. The fully quantum mechanical CDCC model NCBU observables are shown by the solid curves. Those from the classical model using the same breakup probability function are quite similar (dashed curves). Using an empirical $\mathcal{P}_{\mathrm{BU}}^{L}(R)$ deduced from the ${ }^{9} \mathrm{Be}+{ }^{208} \mathrm{~Pb}$ reaction gives very different results (dotted curves), which are scaled by 0.1 where noted, demonstrating the sensitivity of the observables to $\mathcal{P}_{\mathrm{BU}}^{L}(R)$. 


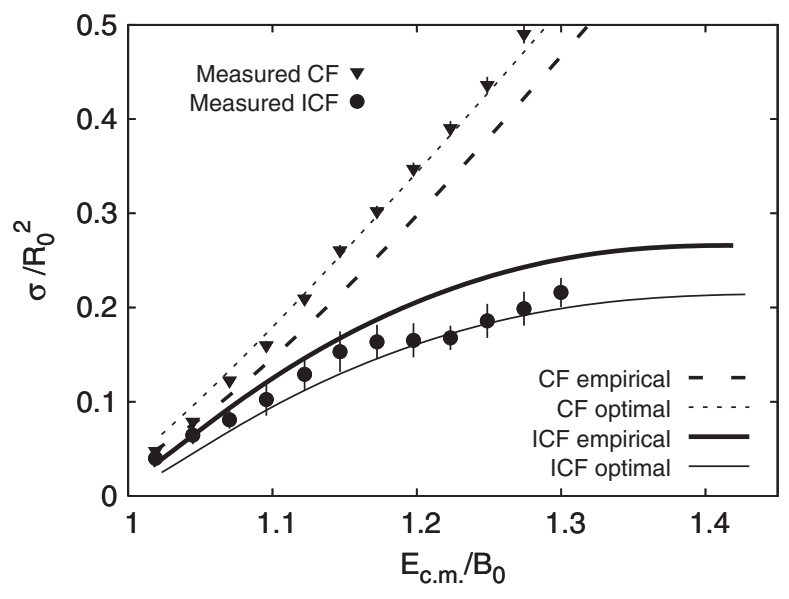

FIG. 3. The classical model CF (dashed curves) and ICF (solid curves) cross sections for ${ }^{8} \mathrm{Be}+{ }^{208} \mathrm{~Pb}$ are compared with the ${ }^{9} \mathrm{Be}+{ }^{208} \mathrm{~Pb}$ experimental data of Ref. [1]. The energy and the cross section of the calculated and experimental data are normalized by the barrier energies $\left(B_{0}=40.7 \mathrm{MeV}\right.$ for thick curves, $B_{0}=40.5 \mathrm{MeV}$ for thin curves, and $B_{0}=37.5 \mathrm{MeV}$ for experimental data) and their respective radii $\left(R_{0}=10.7 \mathrm{fm}\right.$ for theoretical curves and $R_{0}=10.8 \mathrm{fm}$ for experimental data), obtained by fitting the above-barrier total fusion (CF + ICF) cross sections with the formula $\sigma_{\text {fus }}=\pi R_{0}^{2}\left(1-B_{0} / E_{\text {c.m. }}\right)$.

quite well, both qualitatively and quantitatively, providing support for the classical dynamical treatment.

Figure 2 also shows the strong sensitivity of the (classical model) observables to the breakup function assumed. The dotted curves result from using the empirical $\mathcal{P}_{\mathrm{BU}}^{L}(R)$ from the ${ }^{9} \mathrm{Be}+{ }^{208} \mathrm{~Pb}$ experiment, as opposed to the CDCC breakup function (dashed curve). The former results in breakup occurring much nearer to $R_{\min }$ and thus to the target. The NCBU spin distribution when using the empirical breakup function [panel (a)] now peaks at smaller angular momenta with a corresponding shift in the peak of the $\alpha$ angular distribution to larger angles [panel (b)]. The strong tidal forces experienced by the nonfused $\alpha$ particles then lead to an enhanced $\alpha$ - $\alpha$ yield of high relative energy [panel (c)] and corresponding changes to the singles $\alpha$ energies [panel (d)].

These first results already indicate a potentially very useful and very sensitive mapping between (the unobservable) $\mathcal{P}_{\mathrm{BU}}^{L}(R)$ and the NCBU observables shown in Fig. 2. The breakup function and reaction dynamics, probed by combining the classical model and experimental data, could thus provide much needed insight into the localization and nature of the breakup and fusion mechanism for different reacting systems.
Finally, we apply these ideas to experimental data. Using $\mathcal{P}_{\mathrm{BU}}^{L}(R)$ deduced from the empirical breakup function (thick solid line in Fig. 1), the model predicts $\mathrm{CF}$ and ICF yields as shown by the thick solid and dashed curves in Fig. 3. These are close to the experimental data (triangles and circles). Only a small change in $\mathcal{P}_{\mathrm{BU}}^{L}(R)$ is required to give a very good reproduction of the data (thin lines in Figs. 1 and 3). This result is the first quantitative result supporting the suggested linkage [10] between the breakup process observed below the barrier and suppression of complete fusion observed at energies above the barrier.

In summary, a three-dimensional classical dynamical model for low energy reactions of weakly bound nuclei is presented, which for the first time allows consistent realistic calculations relating below-barrier NCBU with above-barrier $\mathrm{CF}$ and ICF. In the test case studied, the model, which treats the breakup process stochastically, is shown to reproduce the differential NCBU observables of the CDCC quantum approach quite accurately. We have shown that the breakup probability function $\mathcal{P}_{\mathrm{BU}}^{L}(R)$ has high diagnostic value, being sensitive to the reaction mechanism and reactants, and that NCBU reaction observables are strongly sensitive to and can interrogate this model input. The model also demonstrates the importance of the breakup probability function, accessible from experiments at sub-barrier energies, to predictions of complete and incomplete fusion yields of weakly bound projectiles at energies above the Coulomb barrier.

All authors acknowledge support from an ARC Discovery grant while J. A. T. acknowledges support also from the United Kingdom Engineering and Physical Sciences Research Council (EPSRC) under Grant No. EP/D003628.

[1] M. Dasgupta et al., Phys. Rev. Lett. 82, 1395 (1999); Phys. Rev. C 70, 024606 (2004).

[2] P. R. S. Gomes et al., Phys. Lett. B 634, 356 (2006).

[3] K. Hagino et al., Phys. Rev. C 61, 037602 (2000).

[4] A. Diaz-Torres et al., Phys. Rev. C 65, 024606 (2002); 68, 044607 (2003).

[5] K. Yabana et al., Nucl. Phys. A738, 303 (2004).

[6] I. J. Thompson et al., Prog. Theor. Phys. Suppl. 154, 69 (2004).

[7] J. A. Tostevin et al., Phys. Rev. C 63, 024617 (2001).

[8] K. Hagino et al., Nucl. Phys. A738, 475 (2004).

[9] D. G. Arbo et al., Phys. Rev. Lett. 96, 143003 (2006).

[10] D. J. Hinde et al., Phys. Rev. Lett. 89, 272701 (2002).

[11] G. D. Dracoulis et al., J. Phys. G 23, 1191 (1997).

[12] A. Diaz-Torres et al., Phys. Lett. B 533, 265 (2002). 